\title{
Towards virtual doctor consultations: A call for the scale-up of telemedicine in sub-Saharan Africa during COVID-19 lockdowns and beyond
}

\author{
Itai Chitungo $^{\mathrm{a}}$, Malizgani Mhango ${ }^{\mathrm{b}}$, Mathias Dzobo ${ }^{\mathrm{a}}$, Knowledge Denhere ${ }^{\mathrm{c}}$, \\ Munashe Chimene $^{\mathrm{d}}$, Godfrey Musuka ${ }^{\mathrm{e}}$, Tafadzwa Dzinamarira ${ }^{\mathrm{f},}$ \\ ${ }^{a}$ Department of Diagnostic Medicine and Investigative Sciences, Faculty of Medicine and Health Sciences, University of Zimbabwe, Harare, Zimbabwe \\ ${ }^{\mathrm{b}}$ School of Public Health, University of Western Cape, 7535, Cape Town, South Africa \\ ${ }^{\mathrm{c}}$ School of Public Health, University of Western Cape, South Africa \\ ${ }^{\mathrm{d}}$ COVID-19 Response Team, Chinhoyi Provincial Hospital, Chinhoyi, Zimbabwe \\ e ICAP@ Columbia University, Harare, Zimbabwe \\ ${ }^{\mathrm{f}}$ Department of Public Health Medicine, School of Nursing and Public Health, University of KwaZulu-Natal, Durban, 4001, South Africa
}

\section{A R T I C L E I N F O}

\section{Keywords:}

Telemedicine

COVID-19

Sub-Saharan Africa

Telehealth

Scale-up

\begin{abstract}
A B S T R A C T
The outbreak of COVID-19 has resulted in adoption and implementation of mitigatory policies, including movement restrictions (lockdowns) to curb its spread. These lockdowns have brought unintended consequences such as increasing the inequalities in health delivery. In the context of these restrictions, telemedicine provides an opportunity for continuation of essential health care provision. This review aimed to map available literature on the current status of telemedicine in sub-Saharan Africa to proffer recommendations for scale up during COVID-19 and beyond. Our review highlighted the lack of meaningful investment in the area. The literature identified resistance to telemedicine, infrastructural barriers, and the lack of policy and budgetary support as main deterrents to current implementation. We recommend the region to leverage on the rapid expansion of internet and telecommunication in addition to adopting a mix of strategies to set up an infrastructure for providing scale up of telemedicine and overcome barriers to implementation. There is an urgent need for policy formulation and the provision of budgetary support through sustainable business models.
\end{abstract}

\section{Introduction}

The emergence of pneumonia of unknown etiology in Wuhan in December 2019 which was declared a public health emergency by the World Health Organisation (WHO) has stirred global public health concerns (WHO, 2020). The causative agent of the pneumonia was eventually attributed to a member of the coronavirus family on the January 7, 2020 and the WHO named the disease coronavirus disease (COVID-19) on the February 11, 2020 (Rothan \& Byrareddy, 2020; Zhou et al., 2020). The possibility of human to human spread coupled with the rapid rise in global cases forced nations to adopt and implement restrictive measures such as social distancing, quarantining of the affected and at-risk individuals, closure of borders, and lockdown of cities to mitigate the spread of the virus (Lai,

\footnotetext{
* Corresponding author. Department of Public Health Medicine, School of Nursing and Public Health, University of KwaZulu-Natal, Durban, 4001, South Africa.

E-mail address: anthonydzina@gmail.com (T. Dzinamarira).
} 
Shih, Ko, Tang, \& Hsueh, 2020). Despite the pandemic mitigating efforts, unintended consequences such as increasing the inequalities in health delivery and other disparities were observed in low-to medium income countries (LMICs) (Sonu Bhaskar, Sian Bradley, et al., 2020; Dzinamarira, Dzobo, \& Chitungo, 2020; Mhango, Chitungo, \& Dzinamarira, 2020). For instance, millions of people in Africa lack access to hygiene facilities and clean water services. This has threatened the ability to curb the spread of COVID-19 given the importance of handwashing in COVID-19 control (Okoi \& Bwawa, 2020).

Sub-Saharan Africa (SSA) with a population close to a billion is constantly troubled by infectious diseases. It has a low physician-topopulation ratio of about 1:30,000, compared to 1:300 in the developed world constraining health delivery and mostly disadvantaging populations in rural and remote areas (Bouskill, Smith-Morris, Bresnick, Cuadros, \& Pedersen, 2018; Combi, Pozzani, \& Pozzi, 2016). The pandemic disrupted livelihood of the continent majority who cannot afford to follow evidenced based social distance policies, have limited access to COVID-19 testing and care facility. Additionally, the already under service remote areas are further disadvantaged by imposed travel restrictions. Those living with chronic illness are afraid to visit healthcare centres due to fears of contracting COVID-19 (Sonu Bhaskar, Sian Bradley, et al., 2020). Another consequence of the pandemic was cancellation of clinical rotations, causing loss critical learning opportunities (Li \& Bailey, 2020).

However, SSA as a region remains the fastest growing market for smartphone and internet connectivity (Edo, Okodua, \& Odebiyi, 2019). Technology can be transformational and helps willing governments to promote the inclusion of disadvantaged groups. Through investing in digital infrastructure that expands internet connectivity and improve power supply using a mix of market competition, private-public partnership and effective regulation of internet and telecom sector (Bank, 2016). SSA governments can provide concrete opportunities to tackle health system challenges and thereby offer the potential to enhance the coverage and quality of health practices and services. Digital health solutions can assist HCW with immediate access to clinical protocols, decision support mechanism or teleconsultations with other health workers strengthening health systems and improve care (WHO guideline-recommendation on digital interventions for health system strengthening (WHO, 2019). Tele-education has potential in LMIC to offer an attractive option to accelerate knowledge translation and address infrastructure barriers and limited opportunities of subspecialty training and significantly improve clinical outcomes and associated costs (Kovacevic et al., 2019). To fully harness the benefits of telemedicine it must be appropriately integrated into the health delivery. The rapid technological advancements have revolutionized the medical landscape, allowing its expansion out of the traditional healthcare facility such as consultation rooms and clinics and overcoming barriers posed by geographical locations (Srinivasan et al., 2020) making it feasible to reduce inequalities in health delivery. Telemedicine has the potential to address the current the inequalities resulting from the COVID-19 pandemic and countermeasures to contain it, thus strengthen the continent's constrained health delivery system during and after COVID-19. This review aimed to map available literature on the current status of telemedicine in sub-Saharan Africa to proffer recommendations for scale up during COVID-19 and beyond.

\section{Methodology}

We conducted a narrative literature review on telemedicine in SSA. The review also searched for evidence on access to health care services during the COVID-19 pandemic across SSA countries. During the period December 14th - 18th 2020, we searched for articles published in English on the WHO website, peer reviewed articles on Google Scholar and PubMed, official public health websites operated by the governments, and newspaper articles primarily written from within SSA. We used keywords that included COVID-19; response; Africa; telemedicine; telehealth. The first and second authors reviewed the literature from the bibliography and drafted the first draft of the paper. The articles listed on our reference list are those quoted directly and do not necessarily represent the sources reviewed. Consistent with narrative review methodology, some steps such as quality appraisal of the evidence that would occur with a systematic review were omitted.

\section{Current state of the COVID-19 pandemic in sub Saharan Africa}

At the time of writing (December 2020), there were over 4,3 million confirmed COVID-19 cases globally with over 1,5 million deaths. At the same time, over 1,6 million cases and over in Africa cases and 1,599,922 global deaths with Africa contributing 35,000 deaths (WHO, 2021). At the same time of writing, most African countries were experiencing a second wave of COVID- 19 infections. Most governments implemented another round of restrictive measure that limited travel, resulting in unwanted limited access to health services. The continent was also affected by severe shortages of personal protective equipment (PPE) creating unsafe working for HCWs. Alternative medical supply chain and health service delivery models are urgently needed to address health service access challenges (Sonu Bhaskar, Jeremy Tan, et al., 2020).

\section{Current state of TM in sub Saharan Africa}

The World Health Assembly in 2005 promulgated a resolution urging member countries to put in place strategies for the development of TM and to achieve coverage for all by 2015 as part of millennium development goals (Ryu, 2012). This section will discuss some of the major TM projects in the previous decade. The University Hospital Geneva from 2000 introduced the Réseau en Afrique Francophone pour la Télémédecine (RAFT) project in more than 10 African counties that involved tele consulting, telepathology and tele-education (Geissbuhler, Bagayoko, \& Ly, 2007). The first country for RAFT implementation was Mali and in 2003 the government initiated an e Health project ANTIM that was integrated with RAFT and pesinet for telemedicine reach across the country (Geissbuhler, Ly, Lovis, \& L'Haire, 2003). EuropeAid between 2009 and 2011 rolled out Eb@le-Santé project at four academic hospitals to improve 
health care through easy access to electronic records and teleeducation. Other telemedicine projects in the last decade where in Zambia, Ethopia, Eygpt, and Ghana (Combi et al., 2016). Recently, African members states are instituting telemedicine project such as the Angosat in Angola, mTrac in Uganda, and Ghana is setting up teleconsulting centres across the country (Oluwakemi, 2020).

\section{Barriers to full scale implementation of TM}

The current barriers to implementation of TM in SSA are presented in Table 1. The literature identified resistance to telemedicine, infrastructural barriers, and the lack of policy and budgetary support as main deterrents to current implementation.

\subsection{Resistance to $T M$}

Global uptake of TM has been limited especially in Africa due to myriad reasons (Mars, 2013). Barring limitations, Ethiopia and South Africa tried to implement TM programs in their respective countries, but this met resistance and limited buy-in from practitioners (Townsend \& Scott, 2019). Anthropological and cultural dynamics such as user acceptance of newer technologies by patients and doctors, inspiration, training, and staff support affect use of TM. Regardless of the increasing reception of TM, some patients still prefer face to face consultations (Albarrak et al., 2019; Bele et al., 2019; Farid, 2019; Ggita et al., 2018; Ray, Demirci, Bogen, Mehrotra, \& Miller, 2015). TM involves substantial modifications to the current workflows and many HCWs and providers would have to invest time in training, adjust to new workflows and systems, and this disturbs both effectiveness and efficacy of health services (Bhaskar et al., 2020a). The current pandemic has already disrupted healthcare delivery and both HCWs and patients should embrace TM as it is a safe, effective and efficient approach to health delivery. Training can be achieved through collaboration of governments and learning institutions and professional bodies to impact skills to HCW and develop learning strategies as well as coaching patients the technological skills necessary to access TM (Bigna, Noubiap, Plottel, ). Finally, it is worth noting language barriers may contribute to resistance to telemedicine uptake (Sagaro, Battineni, \& Amenta, 2019; Zobair, Sanzogni, \& Sandhu, 2020). There is need for linguistically sensitive telemedicine platforms appropriate to specific populations.

\subsection{Infrastructure hurdles to TM implementation}

The high cost of infrastructure development and maintenance is a limiting barrier to TM globally. This also translate to high cost of broadband which limits the dissemination of telemedicine in SSA (Watts \& Abraham, 2020). Maintenance issues including hardware replacement, software upgrades, and equipment failures must be attended to instantly by a qualified and proficient engineer. Although few problems can be fixed by distant troubleshooting processes, equipment failure often require on-site visits. In SSA, paucity of skilled and qualified engineers makes this assignment challenging and expensive. The capacity of having distant monitoring tools and technologies that enables off-site maintenance can considerably decrease costs and reduce interruptions (Hollander \& Carr, 2020). However, SSA as a region remains the fastest growing market for smartphone and internet connectivity. Technology can be transformational and helps willing governments to promote the inclusion of disadvantaged groups through investing in digital infrastructure that expands internet connectivity and improve power supply using a mix of market competition, private-public partnership and effective regulation of internet and telecom sector. SSA governments can provide concrete opportunities to strengthen health systems (Bouskill et al., 2018; Sims, 2018). The pandemic has shown the capacity of global partnerships which the continent can leverage to create partnership with manufacturers of TM equipment, this will capacitate the continent by providing requisite training to local engineers and therefore, creating employment and robust service back up.

Table 1

Presentation of current barriers to implementation of telemedicine in SSA and recommendations for scale-up.

\begin{tabular}{|c|c|c|}
\hline Barriers & Recommendations for scale-up & Future/long term considerations \\
\hline $\begin{array}{l}\text { 1. Health worker-related barriers } \\
\text { - Persistent health workforce shortages, } \\
\text { compounded by COVID-19 related morbidity } \\
\text { and mortality among health workers } \\
\text { - Lack of health care worker buy-in } \\
\text { - Limited technological know-how } \\
\text { - Language barriers }\end{array}$ & $\begin{array}{l}\text { - Utilize virtual consultations for asymptomatic } \\
\text { doctors unable to be physically present at hospitals } \\
\text { due to COVID-19 isolation or quarantine. } \\
\text { - Redesign health care workers' workload } \\
\text { incorporating telemedicine } \\
\text { - Mass technology training for both patients and } \\
\text { health care workers } \\
\text { - Incorporate different linguistics in TM relevant to } \\
\text { the target population }\end{array}$ & $\begin{array}{l}\text { Educational reforms that improve } \\
\text { technological knowledge and inclusion } \\
\text { of telemedicine training }\end{array}$ \\
\hline $\begin{array}{l}\text { 2. Infrastructure hurdles } \\
\text { - High cost of set up } \\
\text { - Maintenance skills gap }\end{array}$ & $\begin{array}{l}\text { - Global partnerships with manufacturers of } \\
\text { equipment } \\
\text { - Support private-public partnerships to unlock in- } \\
\text { vestment in TM infrastructure }\end{array}$ & $\begin{array}{l}\text { Training or upskilling local engineers } \\
\text { to attend to maintenance issues }\end{array}$ \\
\hline $\begin{array}{l}\text { 3. Lack of policy and budgetary support } \\
\text { - Lack of policy clarity } \\
\text { - Limited budgetary support }\end{array}$ & $\begin{array}{l}\text { - Development of national policy on TM with } \\
\text { sufficient devolution } \\
\text { - Increase budgetary support to health and } \\
\text { specifically TM }\end{array}$ & $\begin{array}{l}\text { Consistent policy and budgetary } \\
\text { support }\end{array}$ \\
\hline
\end{tabular}




\subsection{Lack of policy and budgetary support}

Policy barriers coupled with limited budgetary support for devolution hinder the smooth operation, expansion and development of any system. Definite guidelines and procedures at national level are necessary for TM to gain traction in any country. These welldefined guidelines, protocols, procedures, and proprieties are essential to help a TM system to run efficiently and safely and guarantee that populations are receiving quality healthcare services. Most developing countries including SSA lack uniform and regular TM policies, which creates confusion in designing TM-related services, programs, and even its implementation (Bali, 2018a, 2018b).

The rapid scale-up of TM during the COVID-19 pandemic may have long-term implications for access to both general and subspecialty care in SSA. As more health providers are trained and more facilities equipped with TM infrastructure, these services will likely continue to be offered beyond the pandemic (Beller et al., 2020). TM may offer several benefits for health systems in SSA including providing expanded access to services, fostering the integration of rural primary care and remote subspecialty services, and enabling more effective care management (Bali, 2018a). In order for TM to be effectively implemented in SSA, coverage and reimbursement, licensure, internet access, and privacy and security will need to be adequately addressed. Improved access to subspecialty care via TM may be an unexpected consequence of the COVID-19 pandemic. Granted that SSA will not have initial capacity to fully integrate TM and implement it, countries can utilize existing technologies and build capacity over time through policy and budgetary support, sustainable business models and training.

\section{Discussion}

The case to scale up telemedicine for SSA during COVID-19 lockdowns and beyond.

Table 1 presents recommendations for the scale up of TM in SSA. Research has revealed TM success in breaking geographical and time hurdles experienced in the receipt of healthcare in traditional modalities with similar or even superior efficiency (Scott Kruse et al., 2018). TM increases accessibility to routine and specialised medical services, delivers healthcare to remote rural areas, provides HCWs with better flexibility in patient scheduling, and saves clients' time and money in seeking care. TM helps to enforce social distancing and minimise person to person contact (Cooley, 2020) as per WHO recommendation. TM enables forward triaging of patients, helping to segregate confirmed or clients at high risk for COVID-19 from those with underlying conditions such as diabetes mellitus, cardiac problems or asthma (Smith et al., 2020). Furthermore, the adoption of TM will decongest healthcare centres and allow the general population to continue accessing safe and convenient quality care regardless of COVID-19 exposure (Barba et al., 2020). Under COVID-19 lockdown TM has the potential to create linkages between ICU experts in developed countries and clinicians attending to a patient in resource-limited settings, thus, improving health outcomes. Due to the need created by the COVID-19 outbreak, developed countries experienced a surge in uptake and use of TM amid social distancing and stay-at-home directives. Concerns about both HCW and general population safety in combination with urgent regulatory changes have been the main driving forces behind the rapid adoption of TM (Poppas, Rumsfeld, \& Wessler, 2020).

TM offers SSA the opportunity to collect and organize patient data for epidemiological surveillance and assist in public health response (Macabasag, Magtubo, \& Marcelo, 2016; Portnoy, Waller, \& Elliott, 2020). China, Australia, Brazil and the United Kingdom are using app-facilitated screening, triaging and tracing of their populations which have improved the efficiency and effectiveness of the traditional healthcare setting. Data collected from these platforms make it possible to identify COVID-19 hotspots and populations at high risk, thus achieving a better understanding of the disease and allowing for a more coordinated and better patient follow up and evaluation (Smith et al., 2020). While, TM is unable to accurately predict natural disasters or infectious pandemics, it surely gives an advantage towards preparing for such eventualities and therefore, timeous response.

Amid an increase in chronic and non-communicable diseases in Africa, SSA can benefit by employing TM as evidenced prior to COVID-19 in the USA, whereby TM solutions in the management of chronic diseases improved the total cost of care by $2-3$ percent. TM can provide rapid access to specialists who are not physically available to clients in remote settings who are usually financially constrained and limited in their mobility to access quality healthcare (Ray et al., 2015). The system leverages specialists at distant sites to provide teleconsultations for emergencies and subspecialties. Furthermore, TM can be used to provide on-demand training or remote training (Watts \& Abraham, 2020). TM will help capacitate HCWs through quick access to clinical manuals, decision support mechanism or teleconsultations with other health workers hence strengthening health systems and improving care. Tele-education in LMIC offers an attractive option to accelerate knowledge translation and overcome infrastructural barriers and subspecialty shortages which will significantly improve clinical outcomes and decrease healthcare costs. Additionally, technologies for remote education and training delivery allow for continued learning for healthcare workers (Li \& Bailey, 2020). There is need for telemedicine-based technologies to maintain continuity in medical education; especially when the traditional education has been severely affected, more so in under-resourced settings (Sharma \& Bhaskar, 2020).

To fully harness the benefits of telemedicine, it must be appropriately integrated into the health delivery (Bhaskar et al., 2020b; Smith et al., 2020). The WHO encourages the creation of national agencies that facilitate, contextualize and review telemedicine adoption and use based on local capacity and needs.

\section{Conclusion}

The primary thrust of governments is to reign in the COVID-19 pandemic. African governments imposed restrictive measures including social distancing to prevent healthcare system overcrowding and depletion of medical supplies and resources. However, these restrictive measures have caused inequalities to healthcare accesses. TM systems are ideal for mitigating COVID-19 spread and 
decongest healthcare facilities allowing for quality care delivery. The literature identified resistance to telemedicine, infrastructural barriers, lack of policy and budgetary support as main deterrents to implementation. The continent can leverage on the rapid expansion of internet and telecommunication in addition to adopting a mix of strategies to set up an infrastructure for providing care using TM and overcome barriers to implementation. There is an urgent need for policy formulation and the provision of budgetary support through sustainable business models.

\section{Credit author statement}

Itai Chitungo; Malizgani Mhango; Mathias Dzobo: Conceptualization, Methodology; Formal analysis, Writing - original draft, Writing - review \& editing, Knowledge Denhere; Dr. Munashe Chimene: Writing - review \& editing. Dr. Godfrey Musuka; Dr. Tafadzwa Dzinamarira: Supervision, Writing - review \& editing.

\section{Declaration of competing interest}

The authors declare that they have no known competing financial interests or personal relationships that could have appeared to influence the work reported in this paper.

\section{References}

Albarrak, A. I., Mohammed, R., Almarshoud, N., Almujalli, L., Aljaeed, R., Altuwaijiri, S., et al. (2019). Assessment of physician's knowledge, perception and willingness of telemedicine in Riyadh region, Saudi Arabia. Journal of Infection and Public Health.

Bali, S. (2018a). Barriers to development of telemedicine in developing countries. In Telehealth. IntechOpen.

Bali, S. (2018b). Enhancing the reach of health care through telemedicine: Status and new possibilities in developing countries. Health care delivery and clinical science: Concepts, methodologies, tools, and applications (pp. 1382-1397). IGI Global.

Bank, W. (2016). World development report 2016: Digital dividends. Available from: https://www.worldbank.org/en/publication/wdr2016. (Accessed 1 March 2021).

Barba, R., Rosado, C., Pardo-Moreno, J., \& Rey-Biel, J. (2020). Managing people, roles, and resources during covid-19 surge how the Rey Juan carlos university hospital in Madrid quickly modified its spaces, staffing levels, and roles to confront a surge of covid-19 patients. Retrieved from https://catalyst.nejm.org/doi/ full/10.1056/cat.20.0152.

Bele, S., Cassidy, C., Curran, J., Johnson, D. W., Saunders, C., \& Bailey, J. M. (2019). Barriers and enablers to implementing a virtual tertiary-regional telemedicine rounding and consultation (TRAC) model of inpatient pediatric care using the theoretical domains framework (TDF) approach: A study protocol. BMC Health Services Research, 19(1), 1-9.

Beller, H. L., Rapp, D. E., Noona, S. W., Winkelman, A. J., Zillioux, J. M., Smith, R. P., ... Greene, K. L. (2020). Tele-urology during COVID-19: Rapid implementation of remote video visits. Urology Practice, 10.1097/UPJ. 0000000000000167.

Bhaskar, S., Bradley, S., Chattu, V. K., Adisesh, A., Nurtazina, A., Kyrykbayeva, S., ... Thomas, P. (2020a). Telemedicine across the globe-position paper from the COVID-19 pandemic health system resilience PROGRAM (REPROGRAM) international consortium (Part 1). Frontiers in Public Health, 8.

Bhaskar, S., Rastogi, A., Menon, K. V., Kunheri, B., Balakrishnan, S., \& Howick, J. (2020b). Call for action to address equity and justice divide during COVID-19. Frontiers in Psychiatry, 11, 559905. https://doi.org/10.3389/fpsyt.2020.559905

Bhaskar, S., Tan, J., Bogers, M. L. A. M., Minssen, T., Badaruddin, H., Israeli-Korn, S., et al. (2020c). At the epicenter of COVID-19-the tragic failure of the global supply chain for medical supplies. Frontiers in Public Health, 8. https://doi.org/10.3389/fpubh.2020.562882, 562882-562882.

Bigna, J. J. R., Noubiap, J. J. N., Plottel, C. S., Kouanfack, C., \& Koulla-Shiro, S. (2014). Barriers to the implementation of mobile phone reminders in pediatric HIV care: A pre-trial analysis of the Cameroonian MORE CARE study. BMC Health Services Research, 14(1), 523.

Bouskill, K., Smith-Morris, C., Bresnick, G., Cuadros, J., \& Pedersen, E. R. (2018). Blind spots in telemedicine: A qualitative study of staff workarounds to resolve gaps in diabetes management. BMC Health Services Research, 18(1), 617.

Combi, C., Pozzani, G., \& Pozzi, G. (2016). Telemedicine for developing countries. Applied Clinical Informatics, 7(4), 1025-1050.

Cooley, L. (2020). Fostering human connection in the covid-19 virtual health care realm. Retrieved from https://catalyst.nejm.org/doi/full/10.1056/CAT.20.0166? query=CON\&cid=DM92433_Catalyst_Non_Subscriber\&bid=204343456.

Dzinamarira, T., Dzobo, M., \& Chitungo, I. (2020). Covid-19: A perspective on Africa's capacity and response. Journal of Medical Virology.

Edo, S., Okodua, H., \& Odebiyi, J. (2019). Internet adoption and financial development in sub-saharan Africa: Evidence from Nigeria and Kenya. African Development Review, 31(1), 144-160.

Farid, S. F. (2019). Conceptual framework of the impact of health technology on healthcare system. Frontiers in Pharmacology, 10, 933.

Geissbuhler, A., Bagayoko, C. O., \& Ly, O. (2007). The RAFT network: 5 years of distance continuing medical education and tele-consultations over the internet in French-speaking Africa. International Journal of Medical Informatics, 76(5-6), 351-356.

Geissbuhler, A., Ly, O., Lovis, C., \& L'Haire, J.-F. (2003). Telemedicine in Western Africa: Lessons learned from a pilot project in Mali, perspectives and recommendations. In Paper presented at the AMIA annual symposium proceedings.

Ggita, J. M., Ojok, C., Meyer, A. J., Farr, K., Shete, P. B., Ochom, E., et al.Dowdy, D. (2018). Patterns of usage and preferences of users for tuberculosis-related text messages and voice calls in Uganda. International Journal of Tuberculosis \& Lung Disease, 22(5), 530-536.

Hollander, J. E., \& Carr, B. G. (2020). Virtually perfect? Telemedicine for covid-19. New England Journal of Medicine, 382(18), 1679-1681. https://doi.org/10.1056/ NEJMp2003539

Kovacevic, P., Dragic, S., Kovacevic, T., Momcicevic, D., Festic, E., Kashyap, R., et al.Gajic, O. (2019). Impact of weekly case-based tele-education on quality of care in a limited resource medical intensive care unit. Critical Care, 23(1), 1-7.

Lai, C.-C., Shih, T.-P., Ko, W.-C., Tang, H.-J., \& Hsueh, P.-R. (2020). Severe acute respiratory syndrome coronavirus 2 (SARS-CoV-2) and corona virus disease-2019 (COVID-19): The epidemic and the challenges. International Journal of Antimicrobial Agents, 105924.

Li, H. O., \& Bailey, A. M. J. (2020). Medical education amid the COVID-19 pandemic: New perspectives for the future. Academic Medicine, 95(11), e11-e12. https:// doi.org/10.1097/acm.0000000000003594

Macabasag, R. L. A., Magtubo, K. M. P., \& Marcelo, P. G. F. (2016). Implementation of telemedicine services in lower-middle income countries: Lessons for the Philippines. Journal of the International Society for Telemedicine and eHealth, 4(21-11), e24.

Mars, M. (2013). Telemedicine and advances in urban and rural healthcare delivery in Africa. Progress in Cardiovascular Diseases, 56(3), 326-335.

Mhango, M., Chitungo, I., \& Dzinamarira, T. (2020). COVID-19 lockdowns: Impact on facility-based HIV testing and the case for the scaling up of home-based testing services in sub-Saharan Africa. AIDS and Behavior, 1.

Okoi, O., \& Bwawa, T. (2020). How health inequality affect responses to the COVID-19 pandemic in Sub-Saharan Africa. World Development, 135, 105067. https://doi. org/10.1016/j.worlddev.2020.105067

Oluwakemi, S. (2020). Africa needs telemedicine to overcome its healthcare challenges. Published september 16, 2020. Available from: https://africanews.space/ africa-needs-telemedicine-to-overcome-its-medical-challenges/. (Accessed 28 February 2021). 
Poppas, A., Rumsfeld, J. S., \& Wessler, J. D. (2020). Telehealth is having a moment: Will it last? Journal of the American College of Cardiology, 75(23), 2989-2991. https://doi.org/10.1016/j.jacc.2020.05.002

Portnoy, J., Waller, M., \& Elliott, T. (2020). Telemedicine in the era of COVID-19. J Allergy Clin Immunol Pract, 8(5), 1489-1491. https://doi.org/10.1016/j. jaip.2020.03.008

Ray, K. N., Demirci, J. R., Bogen, D. L., Mehrotra, A., \& Miller, E. (2015). Optimizing telehealth strategies for subspecialty care: Recommendations from rural pediatricians. Telemedicine and e-Health, 21(8), 622-629.

Rothan, H. A., \& Byrareddy, S. N. (2020). The epidemiology and pathogenesis of coronavirus disease (COVID-19) outbreak. Journal of Autoimmunity, 102433.

Ryu, S. (2012). Telemedicine: Opportunities and developments in member states: Report on the second global survey on eHealth 2009 (global observatory for eHealth series, volume 2). Healthcare Informatics Research, 18(2), 153-155. https://doi.org/10.4258/hir.2012.18.2.153

Sagaro, G. G. G., Battineni, G., \& Amenta, F. (2019). A review on barriers to sustainable telemedicine implementation in Ethiopia.

Scott Kruse, C., Karem, P., Shifflett, K., Vegi, L., Ravi, K., \& Brooks, M. (2018). Evaluating barriers to adopting telemedicine worldwide: A systematic review. Journal of Telemedicine and Telecare, 24(1), 4-12.

Sharma, D., \& Bhaskar, S. (2020). Addressing the covid-19 burden on medical education and training: The role of telemedicine and tele-education during and beyond the pandemic. Front Public Health, 8, 589669. https://doi.org/10.3389/fpubh.2020.589669

Sims, J. M. (2018). Communities of practice: Telemedicine and online medical communities. Technological Forecasting and Social Change, $126,53-63$.

Smith, A. C., Thomas, E., Snoswell, C. L., Haydon, H., Mehrotra, A., Clemensen, J., et al. (2020). Telehealth for global emergencies: Implications for coronavirus disease 2019 (COVID-19). Journal of Telemedicine and Telecare, $1357633 X 20916567$.

Srinivasan, R., Ben-Pazi, H., Dekker, M., Cubo, E., Bloem, B., Moukheiber, E., et al.Guttman, M. (2020). Telemedicine for hyperkinetic movement disorders. Tremor and Other Hyperkinetic Movements, 10.

Townsend, B., \& Scott, R. (2019). The development of ethical guidelines for telemedicine in South Africa. South African Journal of Bioethics and Law, 12(1), 19-26.

Watts, K. L., \& Abraham, N. (2020). 'Virtually perfect' for some but perhaps not for all: Launching telemedicine in the bronx during the COVID-19 pandemic. In Wolters Kluwer philadelphia, $P A$.

WHO. (2019). Guideline- recommendation on digital interventions for health system strengthening. Geneva World Health Organisation 2019. Available from: https://www. who.int/reproductivehealth/publications/digital-interventions-health-system-strengthening/en/. (Accessed 1 March 2021).

WHO. (2020). Novel coronavirus (COVID-19). World Health Organization. Retrieved from https://www.who.int/emergencies/diseases/novel-coronavirus-2019.

WHO. (2021). COVID-19 in the WHO African region. Retrieved from https://who.maps.arcgis.com/apps/opsdashboard/index.html\#/ 0c9b3a8b68d0437a8cf28581e9c063a9.

Zhou, F., Yu, T., Du, R., Fan, G., Liu, Y., Liu, Z., et al.Gu, X. (2020). Clinical course and risk factors for mortality of adult inpatients with COVID-19 in Wuhan, China: A retrospective cohort study. The lancet.

Zobair, K. M., Sanzogni, L., \& Sandhu, K. (2020). Telemedicine healthcare service adoption barriers in rural Bangladesh. Australasian Journal of Information Systems, 24. 